\title{
Association of postoperative furosemide use with a reduced blood transfusion rate in sagittal craniosynostosis surgery
}

\author{
Adil Harroud, MD, ${ }^{1}$ Alexander G. Weil, MD, FRCSC, ${ }^{1}$ Jean Turgeon, MD, FRCPC, ${ }^{2}$ \\ Claude Mercier, MD, FRCSC, ${ }^{1}$ and Louis Crevier, MD, MSc, FRCSC ${ }^{1}$ \\ ${ }^{1}$ Section of Neurosurgery and 2Department of Pediatrics, CHU Sainte-Justine, Montréal, Québec, Canada
}

OBJECTIVE A major challenge in sagittal craniosynostosis surgery is the high transfusion rate $(50 \%-100 \%)$ related to blood loss in small pediatric patients. Several approaches have been proposed to prevent packed red blood cell (PRBC) transfusion, including endoscopic surgery, erythropoietin or tranexamic acid administration, and preoperative hemodilution. The authors hypothesized that a significant proportion of postoperative anemia observed in pediatric patients is actually dilutional. Consequently, since 2005, at CHU Sainte-Justine, furosemide has been administered to correct the volemic status and prevent PRBC transfusion. The purpose of this study was to evaluate the impact of postoperative furosemide administration on PRBC transfusion rates.

METHODS This was a retrospective study of 96 consecutive patients with sagittal synostosis who underwent surgery at CHU Sainte-Justine between January 2000 and May 2012. The mean age at surgery was $4.9 \pm 1.5$ months (range 2.8-8.7 months). Patients who had surgery before 2005 constituted the control group. Those who had surgery in 2005 or 2006 were considered part of an implementation phase because furosemide administration was not routine. Patients who had surgery after 2006 were part of the experimental (or furosemide) group. Transfusion rates among the 3 groups were compared. The impact of furosemide administration on transfusion requirement was also measured while accounting for other variables of interest in a multiple logistic regression model.

RESULTS The total transfusion rate was significantly reduced in the furosemide group compared with the control group ( $31.3 \%$ vs $62.5 \%$, respectively; $p=0.009$ ), mirroring the decrease in the postoperative transfusion rate between the groups ( $18.3 \%$ vs $50.0 \%$, respectively; $p=0.003$ ). The postoperative transfusion threshold remained similar throughout the study (mean hemoglobin $56.0 \mathrm{~g} / \mathrm{dl}$ vs $60.9 \mathrm{~g} / \mathrm{dl}$ for control and furosemide groups, respectively; $p=0.085$ ). The proportion of nontransfused patients with recorded hemoglobin below $70 \mathrm{~g} / \mathrm{dl}$ did not differ between the control and furosemide groups ( $41.7 \%$ vs $28.6 \%$, respectively; $p=0.489$ ). Surgical procedure, preoperative hemoglobin level, estimated blood loss, and furosemide administration significantly affected the risk of receiving a postoperative PRBC transfusion. When these variables were analyzed in a multiple logistic regression model, furosemide administration remained strongly associated with a reduced risk of being exposed to a blood transfusion (OR 0.196, $p=0.005)$. There were no complications related to furosemide administration.

CONCLUSIONS A significant part of the postoperative anemia observed in patients who underwent sagittal craniosynostosis surgery was due to hypervolemic hemodilution. Correction of the volemic status with furosemide administration significantly reduces postoperative PRBC transfusion requirements in these patients.

http://thejns.org/doi/abs/10.3171/2015.5.PEDS14666

KEY WORDS sagittal synostosis surgery; scaphocephaly; transfusion; furosemide; craniofacial; craniotomy; hemoglobin; blood; sagittal suture; craniosynostosis

$\mathrm{S}$ AGITTAL synostosis is the most common craniosynostosis, affecting approximately 1 of every 2000 newborns. ${ }^{7}$ Surgical correction between 3 and 6 months of age has been shown to achieve the best results. One of the major challenges in this surgery is the associated high rate of packed red blood cell (PRBC) transfusion, with the majority of studies reporting rates between $50 \%$ and $100 \% .{ }^{1,2,-6,9}$ PRBC transfusion carries rare but potentially serious adverse effects related to exposure to foreign antigens and blood-borne pathogens. ${ }^{14}$ Increasing concern

ABBREVIATIONS CBL = calculated blood loss; EBL = estimated blood loss; EBV = estimated blood volume; PRBC = packed red blood cell.

SUBMITTED December 22, 2014. ACCEPTED May 20, 2015.

INCLUDE WHEN CITING Published online October 2, 2015; DOI: 10.3171/2015.5.PEDS14666. 
about these risks has made reducing exposure to homologous blood transfusion a constant priority, particularly in simple craniosynostosis surgery for which the indication is primarily cosmetic. Several approaches have been proposed to prevent homologous blood transfusions, including endoscopic surgery, ${ }^{15}$ erythropoietin ${ }^{8,12,20}$ or tranexamic acid $^{4,9}$ administration, intraoperative autologous blood recycling, ${ }^{20}$ autologous blood transfusion,,${ }^{17}$ preoperative hemodilution, ${ }^{11}$ and accepting a lower transfusion threshold. ${ }^{20}$

The high transfusion rates in sagittal craniosynostosis surgery have been attributed to the small blood volume of the patients coupled with the unavoidable intraoperative blood losses associated with the open procedure. Nevertheless, the early postoperative decline in hemoglobin and hematocrit levels associated with this surgery seemed excessive compared with the observed blood losses and the apparent hemostasis achieved at the end of surgery. We also noticed a considerable postoperative weight gain in these patients, as well as a significant craniofacial edema lasting days after the surgery.

Based on these observations, we hypothesized that in addition to intra- and postoperative blood losses, the anemia observed in these patients was in part due to hypervolemic hemodilution from excessive fluid administration during the anesthesia and subsequent redistribution of the patients' craniofacial interstitial edema toward the intravascular space. Thus, we believed that the postoperative fall in hemoglobin and hematocrit levels was in part artificial and could be managed without resorting to PRBC transfusion. Consequently, in 2005, we implemented a practice of furosemide administration to treat this dilutional component, with the goal of preventing the drop in hemoglobin and reducing postoperative transfusion requirements.

The aim of this study was to retrospectively evaluate the efficacy of furosemide administration for volemic control to reduce postoperative homologous blood transfusions in the setting of sagittal synostosis surgery. The primary outcome variable was reduced postoperative PRBC transfusion.

\section{Methods \\ Patient Population}

This is a retrospective study of patients with sagittal synostosis who underwent surgery at a single institution between January 2000 and May 2012. Institutional review board approval was obtained. Patients were excluded only if craniosynostosis involved an additional suture. A total of 98 patients were eligible for the study. Two patients were subsequently excluded because their charts were incomplete, leaving 96 patients for analysis.

Because postoperative administration of furosemide was first introduced in 2005, patients who underwent surgery prior to 2005 formed our control group $(n=24)$. Patients who had surgery in 2005 or 2006 were considered part of an implementation phase (referred to as the transition group, $\mathrm{n}=12$ ), because postoperative administration of furosemide existed but was not yet routinely used. Patients who underwent surgery after 2006 constituted the experimental group and are referred to as the furosemide group $(n=60)$.

\section{Surgical Procedures}

Patients underwent 1 of 2 surgical procedures performed by 2 neurosurgeons (C.M. and L.C.). Fifty-six patients underwent a standard midline sagittal strip craniectomy measuring $4 \mathrm{~cm}$, with Silastic (Dow Corning) implant and postoperative helmet therapy (Fig. 1 right). The remaining 40 patients underwent an " $\mathrm{H}$ " craniotomy with sagittal osteotomies 2-cm lateral to the sagittal suture and extending anterior to the lambdoid sutures and posterior to the coronal sutures, followed by greenstick fracture of the parietal bones (Fig. 1 left).

\section{Intraoperative Fluid Management}

There was no standard protocol for fluid administration. Patients received mostly crystalloids as a combination of Ringer's lactate and variable concentrations of saline and dextrose. Some patients also received colloids in the form of hydroxyethyl starch (HES), at the discretion of the anesthesiologist. Charts were reviewed to retrieve the volume of intraoperative fluid received by each patient. The total volume was compared between the control and furosemide groups. Because colloids stay within the intravascular space 3 times longer than crystalloids, potentially increasing the probability of hemodilution, we also calculated the mean volume $(\mathrm{ml} / \mathrm{kg})$ of intraoperative fluids received using the following equation: crystalloid volume $(\mathrm{ml} / \mathrm{kg})+3 \times$ colloid volume $(\mathrm{ml} / \mathrm{kg})$.

\section{Blood Loss}

The estimated blood loss (EBL) was assessed by the anesthesiologist and retrieved from the medical records. Calculated blood loss (CBL) is measured based on hemoglobin change from baseline to postoperative level. The formula, developed by Gross, ${ }^{10}$ is intended to provide a more accurate measure of intraoperative blood loss than a physician's estimate. However, because our hypothesis holds that the postoperative hemoglobin is artificially lowered by intraoperative hypervolemic hemodilution, we believe that the CBL may not be ideal for evaluating blood loss in this particular setting. Therefore, it was not included in our analysis. The estimated blood volume (EBV) for these infants was calculated on the basis of $80 \mathrm{ml} / \mathrm{kg}$ of body weight.

\section{Perioperative Management}

All patients had daily monitoring of their hemoglobin, hematocrit, creatinine, and electrolytes values. All patients were weighed preoperatively. Patients who had surgery during or after 2006 also had daily weight recordings until discharge. There was no concomitant administration of erythropoietin or tranexamic acid during the study period. Iron was administered only upon discharge.

In the postoperative period, blood transfusions were given based on the hemoglobin and hematocrit values, the hemodynamic and clinical condition of the infant, as well as the volume status estimated using weight. Hence, hemoglobin values lower than $70 \mathrm{~g} / \mathrm{dl}$ were often toler- 

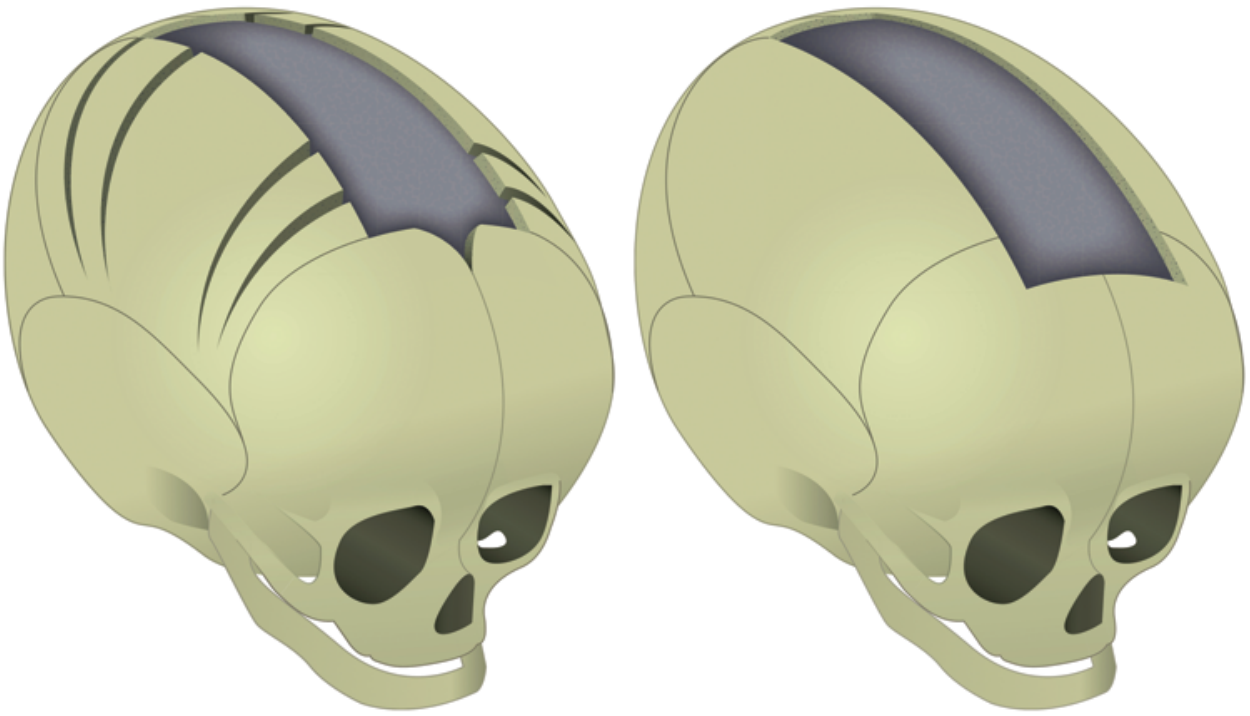

FIG. 1. Schematic representation of the " $\mathrm{H}$ " craniotomy technique (left) and sagittal strip craniectomy (right). Figure is available in color online only.

ated when there were no signs of hemodynamic instability (e.g., hypotension, tachycardia, and tachypnea) and the patient was clinically well.

\section{Furosemide Administration}

Patients in the furosemide group generally received their first dose of furosemide the day after surgery on the basis of $1 \mathrm{mg} / \mathrm{kg}$ of body weight. Some patients received more than 1 dose. Furosemide administered concomitantly to a PRBC transfusion to prevent overload was not considered a dose. The decision regarding furosemide administration was made on an individual basis, taking into account postoperative weight gain, hemoglobin level and trend, and clinical evaluation. The route of administration was intravenous for all patients except for 4 who received an oral form. Electrolytes were closely monitored in all patients who received furosemide.

\section{Postoperative Weight}

The postoperative weight gain was expressed as a proportion of the preoperative weight using the following formula: \%Weight gain $=($ Postoperative Weight - Preoperative Weight)/Preoperative Weight $\times 100 \%$.

\section{Statistical Analysis}

The furosemide and control groups were examined with respect to baseline characteristics, including age, EBL, surgical procedure, operating time, and length of hospital stay. The Student t-test was used to compare the mean lowest hemoglobin level postoperatively between the groups. The rate of intra- and postoperative PRBC transfusion was compared among the 3 predefined groups (i.e., control, transition, and furosemide). The transfusion threshold was compared, as well as the proportion of nontransfused patients with a recorded hemoglobin value $<70$ $\mathrm{g} / \mathrm{dl}$. The impact of several variables on the risk of receiving a postoperative PRBC transfusion was evaluated in a univariate analysis (including age, sex, surgical procedure, operating time, EBL, preoperative weight, preoperative hemoglobin level, and furosemide administration). Potentially confounding variables were included in addition to furosemide administration in a multiple logistic regression model, predicting the probability of receiving a postoperative transfusion of PRBCs.

For all statistical tests, a standard value of $p<0.05$ was considered the criterion for statistical significance. All statistical analyses were conducted using SPSS 20.0 (IBM).

\section{Results}

Ninety-six patients ( 66 boys and 30 girls) were included in the study, with a mean age of $4.9 \pm 1.5$ months (range 2.8-8.7 months). Demographic data and baseline characteristics for each group of patients are presented in Table 1 . The control group had a significantly higher proportion of male patients. In addition, all patients in the control group underwent a strip craniectomy (Fig. 1 right), whereas twothirds of the patients in the furosemide group underwent an " $H$ " craniotomy (Fig. 1 left). The 2 groups were similar with regard to the other variables.

The total PRBC transfusion rate was 15 of 24 patients $(62.5 \%)$ in the control group, 4 of 12 patients $(33.3 \%)$ in the transition group, and 19 of 60 patients $(31.7 \%)$ in the furosemide group. The difference between the control and furosemide groups was statistically significant $(p=0.009)$. The intraoperative transfusion rate was slightly higher in the control group compared with the furosemide group ( $20.8 \%$ vs $13.3 \%$, respectively), but this difference was not statistically significant $(\mathrm{p}=0.505)$. No patient in the transition group received an intraoperative PRBC transfusion. The postoperative PRBC transfusion rate (our primary outcome) dropped from 12 of 24 patients (50\%) in the control group to 4 of 12 (33.3\%) in the transition group and 11 of $60(18.3 \%)$ in the furosemide group (Fig. 2). The difference in postoperative transfusion rates between the control and furosemide groups was statistically significant $(\mathrm{p}=0.003)$. 
TABLE 1. Demographic data and baseline characteristics of the control and furosemide groups

\begin{tabular}{|c|c|c|c|c|}
\hline Variable & $\begin{array}{c}\text { Control Group, } \\
n=24\end{array}$ & $\begin{array}{c}\text { Transition Group, } \\
n=12\end{array}$ & $\begin{array}{l}\text { Furosemide Group, } \\
\qquad n=60\end{array}$ & $p$ Value $^{*}$ \\
\hline Age (mos) $\dagger$ & $4.9 \pm 1.5$ & $4.8 \pm 1.1$ & $5.2 \pm 1.6$ & 0.095 \\
\hline Sex $\neq$ & & & & $0.011 \S$ \\
\hline Male & $21(87.5)$ & $10(83.3)$ & $35(58.3)$ & \\
\hline Female & $3(12.5)$ & $2(16.7)$ & $25(41.7)$ & \\
\hline Surgical technique & & & & $<10^{-3} \S$ \\
\hline Strip craniectomy & $24(100)$ & $12(100)$ & $20(33.3)$ & \\
\hline "H" craniotomy & $0(0)$ & $0(0)$ & $40(66.7)$ & \\
\hline Weight (kg)† & $6.9 \pm 1.0$ & $7.4 \pm 1.4$ & $7.3 \pm 1.2$ & 0.118 \\
\hline Op time $(\mathrm{hrs}) \dagger$ & $2.89 \pm 0.34$ & $2.65 \pm 0.47$ & $2.86 \pm 0.60$ & 0.821 \\
\hline $\mathrm{EBL}(\mathrm{ml}) \dagger$ & $54.6 \pm 27.4$ & $36.8 \pm 18.4$ & $45.0 \pm 17.3$ & 0.122 \\
\hline Preop Hb $(\mathrm{g} / \mathrm{dl}) \dagger$ & $114.4 \pm 9.5$ & $117.9 \pm 10.2$ & $116.9 \pm 10.0$ & 0.290 \\
\hline Preop hematocrit (\%)† & $33.1 \pm 2.8$ & $34.0 \pm 2.8$ & $34.3 \pm 2.6$ & 0.087 \\
\hline \multicolumn{5}{|c|}{$\begin{array}{l}\mathrm{Hb}=\text { hemoglobin. } \\
\text { * Comparing the control and furosemide groups. } \\
\dagger \text { Values are expressed as the median } \pm \text { SD. } \\
\ddagger \text { Values are expressed as no. of patients }(\%) \text {. } \\
\S \text { Statistically significant difference }(p<0.05) \text {, Fisher's exact test. }\end{array}$} \\
\hline
\end{tabular}

The transfusion threshold in the postoperative period was similar throughout the study, with a mean hemoglobin value of $56.0 \pm 5.7 \mathrm{~g} / \mathrm{dl}$ (range $47-65 \mathrm{~g} / \mathrm{dl}$ ) for the control group, $56.2 \pm 5.2 \mathrm{~g} / \mathrm{dl}$ (range $49-62 \mathrm{~g} / \mathrm{dl}$ ) for the transition group, and $60.9 \pm 7.5 \mathrm{~g} / \mathrm{dl}$ (range $49-73 \mathrm{~g} / \mathrm{dl}$ ) for the furosemide group. The proportion of patients who did not receive a postoperative transfusion despite having a hemoglobin value below $70 \mathrm{~g} / \mathrm{dl}$ was 5 of $12(41.7 \%)$ in the control group and 14 of 49 (28.6\%) in the furosemide group. The difference was not statistically significant $(p=0.489)$. The absolute lowest hemoglobin value tolerated in patients who did not receive a blood transfusion postoperatively was $54 \mathrm{~g} / \mathrm{dl}$ in the control group, $54 \mathrm{~g} / \mathrm{dl}$ in the transition group, and $55 \mathrm{~g} / \mathrm{dl}$ in the furosemide group.

A univariate analysis was performed to assess the impact of several variables on the risk of receiving a postoperative transfusion (Table 2). The only variables that correlated with the primary end point were EBL ( $\mathrm{p}=$ $0.022)$, preoperative hemoglobin $(\mathrm{p}=0.025)$, and furo-

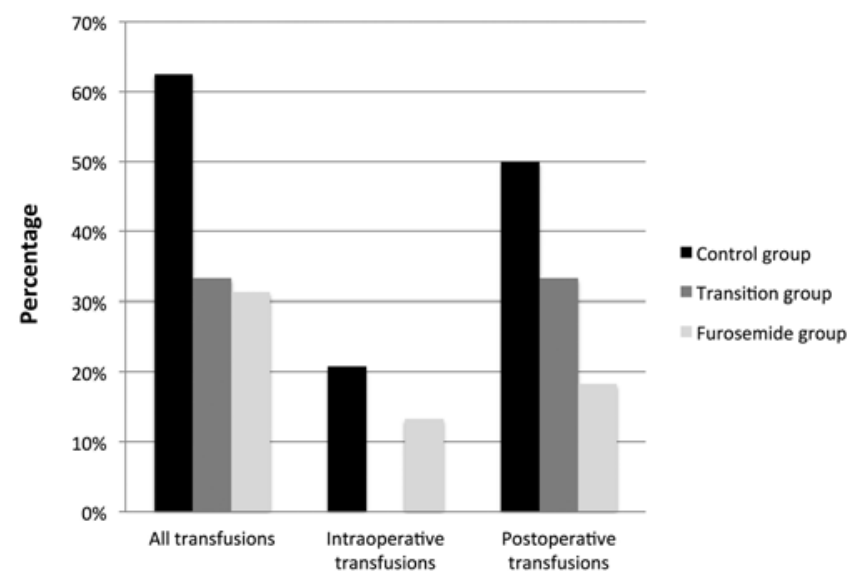

FIG. 2. Histogram showing the percentage of patients in the control, transition, and furosemide groups who received an RBC transfusion. semide administration $\left(\mathrm{p}<10^{-3}\right)$. Age, sex, surgical procedure, operating time, and preoperative weight did not significantly influence the risk of receiving a postoperative transfusion. The variables that significantly impacted the risk of receiving a postoperative transfusion were entered into a multiple logistic regression model (Table 3). Only preoperative hemoglobin $\left(\beta^{2}=0.93, \mathrm{p}=0.030\right)$ and furosemide administration $\left(\beta^{2}=0.19, \mathrm{p}=0.005\right)$ remained significantly correlated with the primary end point.

The data regarding intraoperative fluids received was incomplete or missing for 8 of the 60 patients in the furosemide group. Also, the type of fluid was highly variable in terms of saline and dextrose concentration. The volume of medications received was not available and hence not included in the calculation of total fluid received. Based on the available data, the mean intraoperative fluid volume, combining crystalloids and colloids, received by patients in the control group was $62 \pm 21 \mathrm{ml} / \mathrm{kg}$ compared with $52 \pm 16 \mathrm{ml} / \mathrm{kg}$ in the furosemide group $(\mathrm{p}=0.02)$. However, the proportion of patients who received colloids in the control group was 4 of $24(17 \%)$ compared with 15 of $52(29 \%)$ in the furosemide group. Using the equation to account for the relative 3-fold-greater intravascular component of colloids than crystalloids (Crystalloid volume $[\mathrm{ml} / \mathrm{kg}]+3 \times$ Colloid volume $[\mathrm{ml} / \mathrm{kg}]$ ), the mean intraoperative fluid volume was $66 \mathrm{ml} / \mathrm{kg}$ for the control group compared with $60 \mathrm{ml} / \mathrm{kg}$ for the furosemide group $(\mathrm{p}=$ $0.33)$. The median volume of crystalloids was $50 \mathrm{ml} / \mathrm{kg}$ (range $18-102 \mathrm{ml} / \mathrm{kg}$ ), and the median volume of colloids was $13 \mathrm{ml} / \mathrm{kg}$ (range $6-31 \mathrm{ml} / \mathrm{kg}$ ).

On average, patients gained $6.3 \% \pm 3.0 \%$ of their preoperative weight immediately after surgery and $8.7 \% \pm$ $3.1 \%$ the next day (Fig. 3). The average EBV was $578.9 \pm$ $96.2 \mathrm{ml}$. The average EBL was $43.4 \pm 20.9 \mathrm{ml}$, or $7.5 \%$ of the average EBV. The average postoperative hemoglobin value dropped by $26.4 \% \pm 8.8 \%$ compared with its preoperative level.

Six of 12 patients (50\%) in the transition group received 
TABLE 2. Univariate logistic regression model for predicting postoperative blood transfusion requirement

\begin{tabular}{|c|c|c|c|}
\hline Variable & p Value & OR & $95 \% \mathrm{Cl}$ \\
\hline Age & 0.577 & 0.917 & $0.68-1.24$ \\
\hline Sex ${ }^{*}$ & 0.830 & 0.900 & $0.45-3.12$ \\
\hline Surgical procedure $†$ & 0.055 & 0.382 & $0.14-1.02$ \\
\hline Op time & 0.646 & 0.818 & $0.35-1.92$ \\
\hline EBL & $0.022 \ddagger$ & 1.026 & $1.01-1.05$ \\
\hline Preop weight & 0.273 & 0.811 & $0.54-1.15$ \\
\hline Preop Hb level & $0.025 \neq$ & 0.945 & $0.90-0.99$ \\
\hline Furosemide administration & $<10^{-3} f$ & 0.151 & $0.05-0.40$ \\
\hline
\end{tabular}

at least 1 dose of furosemide. The median number of furosemide doses received was 1 (range $0-3$ doses) and the mean total dose was $9.6 \pm 5.1 \mathrm{mg}$. In the experimental group, 46 of 60 patients (76.7\%) were given furosemide. The 14 patients who did not receive furosemide either: 1) did not have a significant drop in their hemoglobin levels and did not need an intervention $(n=6)$, or 2 ) received an $\mathrm{RBC}$ transfusion intraoperatively $(\mathrm{n}=3)$ or in the recovery room $(n=5)$. Of those who received furosemide, 8 of 46 patients (17.4\%) had a dose the day of surgery; 41 of 46 (89.1\%) 1 day after surgery; 37 of $46(80.4 \%) 2$ days after surgery; 10 of 46 (21.7\%) 3 days after surgery; and only 1 of $46(2.2 \%) 4$ days after surgery. The median number of furosemide doses received was 2 (range 0-6 doses) and the mean total dose was $15.6 \pm 7.6 \mathrm{mg}$. There were no complications related to furosemide administration in either group.

The evolution of hemoglobin levels over days was compared for patients who received furosemide and those who did not, excluding patients who were transfused, to obtain the natural evolution of their postoperative anemia (Fig. 4). Patients who did not receive furosemide had a persistent drop in their hemoglobin levels, especially on Day 3 after surgery. Patients who received furosemide avoided that drop and showed higher hemoglobin levels on Days 3 and 4 after surgery.

\section{Discussion}

Sagittal craniosynostosis surgery has been consistently associated with high transfusion rates of $50 \%-100 \%{ }^{1,2,4-}$ 6,9 The transfusion rate of $62.5 \%$ in our control group is comparable to the rates reported in the literature. Since the introduction of fluid management with postoperative furosemide, total transfusion rates at our institution have been reduced by half during the transition period and for the group of patients receiving furosemide, mainly due to fewer PRBC transfusions during the postoperative period. In fact, the postoperative transfusion rate decreased from $50 \%$ in the control group to $33.3 \%$ in the transition group and $18.3 \%$ in the furosemide group.

The gradual decline in the postoperative transfusion rates from the control group to the transition and furosemide groups parallels the increased use of furosemide at our institution. Furosemide administration was independently associated with a 5 -fold reduction in the risk of being exposed to a blood transfusion, despite a constant transfusion threshold over the course of the study. In addition, the proportion of nontransfused patients with a recorded postoperative hemoglobin value $<70 \mathrm{~g} / \mathrm{dl}$ was similar between the control and furosemide groups. These findings strongly suggest that volemic status correction with postoperative administration of furosemide was responsible for the decrease in transfusion rates without modification of our PRBC transfusion practice.

These findings suggest that furosemide was effective in preventing transfusions through correction of a hypervolemic state responsible for hemodilution and artificially lowered hemoglobin and hematocrit levels. Crystalloid fluids are important during surgery to maintain targeted perfusion pressures while the patient is under general anesthesia.

We believe that the relatively large volumes of crystalloids administered to these young patients, who have a very low EBV, are responsible for hypervolemic hemodilution. In fact, our patients gained an average $8.7 \%$ of their preoperative weight the day after surgery, and the weight gain persisted for several days after surgery. These findings are also consistent with the hypothesis of a hypervolemic state in these patients. Moreover, the EBL

TABLE 3. Multiple logistic regression model for predicting postoperative blood transfusion requirement

\begin{tabular}{llcc}
\hline \multicolumn{1}{c}{ Variable } & $\mathrm{p}$ Value & Adjusted OR & $95 \% \mathrm{Cl}$ \\
\hline Surgical procedure & 0.287 & 0.524 & $0.15-1.65$ \\
\hline EBL & 0.107 & 1.021 & $0.99-1.05$ \\
\hline Preop Hb level & $0.030^{*}$ & 0.941 & $0.89-0.99$ \\
\hline Furosemide administration & $0.005^{*}$ & 0.196 & $0.06-0.61$ \\
\hline
\end{tabular}

* Statistically significant $(p<0.05)$. 


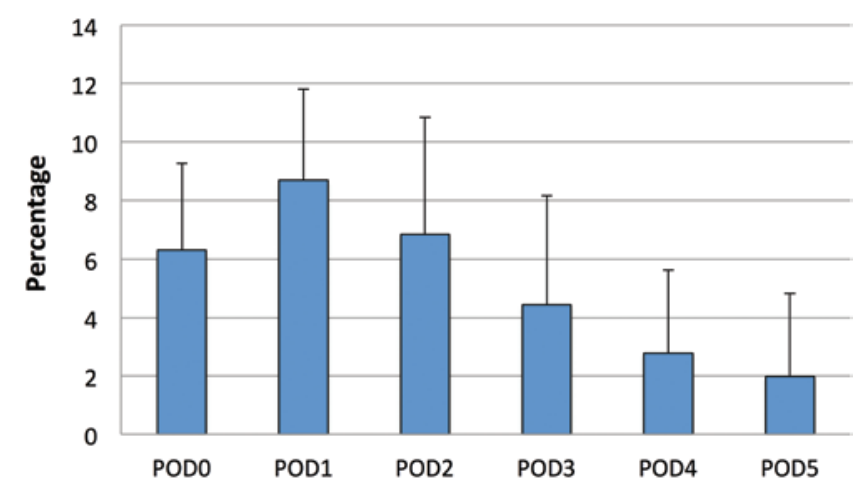

FIG. 3. Histogram showing the postoperative evolution of the mean postoperative weight gain expressed as a percentage of preoperative weight. $\mathrm{POD}=$ postoperative day. Figure is available in color online only.

represented on average $7.5 \%$ of the EBV, while the mean decline in hemoglobin between its preoperative level and immediately after surgery was $26.4 \%$. This important discrepancy suggests that blood loss alone does not explain the observed postoperative anemia and that a significant proportion is secondary to hemodilution.

Patients undergoing sagittal craniosynostosis surgery develop a marked craniofacial edema in the initial days after surgery. This third space seems to start slowly redistributing around the third day after the surgery, contributing to the maintenance of a hypervolemic state. In addition, although these patients usually have a preserved renal function, the postoperative period is characterized by a rise in blood levels of antidiuretic hormone, ${ }^{18}$ which promotes water retention and hemodilution and therefore delays the correction of the volemic status.

The above-discussed hemodilution artificially lowers hemoglobin and hematocrit values and misleads the clinician into giving an unnecessary RBC transfusion. We see a practical advantage in correcting the volemic status with diuretic administration rather than advocating tolerance of very low hemoglobin and hematocrit values, especially considering that we have found the contribution of hemodilution to the postoperative anemia to be very variable. Monitoring of volume overload through weight monitoring and timely administration of furosemide to correct the dilutional component of the postoperative anemia appears to be a feasible alternative.

Previous studies on transfusion rates in craniosynostosis surgery have included patients with heterogeneous pathologies, ranging from single-suture synostosis to complex or syndromic craniosynostosis. ${ }^{21}$ In this study, we present a large and highly homogeneous cohort that includes only patients with sagittal craniosynostosis.

This study has several limitations. Even if administration of furosemide was consistent in the furosemide group, we did not have a clearly defined protocol. The administration of the diuretic and its timing were decided on an individual basis according to the hemoglobin and hematocrit levels, weight gain, and clinical status of the patient, with all patients in the furosemide group with a significant postoperative decrease in hemoglobin levels receiving at least 1 dose. Also, blood loss was based on the estimation of the surgeon and the anesthesiologist. Some studies have

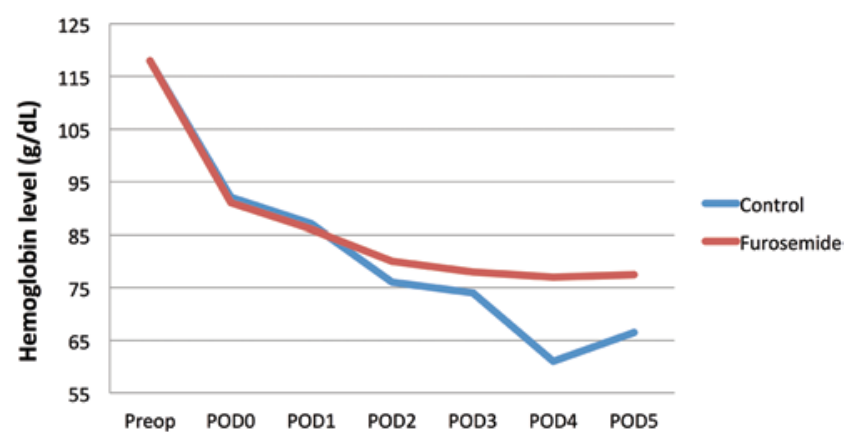

FIG. 4. Graph comparing the evolution of postoperative hemoglobin levels between the control and furosemide groups. Patients who received a PRBC transfusion were excluded. Preop = preoperative level. Figure is available in color online only.

contested the accuracy of this estimation and advocated for use of CBL derived from pre- and postoperative hemoglobin/hematocrit levels and the volume of RBCs transfused. ${ }^{19}$ However, our primary hypothesis was that patients undergoing sagittal craniosynostosis surgery are in a state of hypervolemic hemodilution and that postoperative hemoglobin and hematocrit levels are artificially lowered. Therefore, $\mathrm{CBL}$ was deemed inadequate.

Although there were more " $\mathrm{H}$ " craniotomies in the furosemide cohort, this cannot explain the decreased transfusion rate, because this surgery was associated with a higher blood loss $(50.1 \pm 17.3 \mathrm{ml}$ vs $43.7 \pm 22.9 \mathrm{ml})$. Also, although the implementation of a protocol may have theoretically improved the transfusion rate through furosemide-independent factors, such as increased attention of the surgeon to hemostasis, this was probably not the case because EBL and transfusion threshold did not differ between the furosemide and control cohorts.

Another limitation stemming from the retrospective design of this study is the difference in intraoperative fluid administration. The total amount of fluid received was higher in the control group and could potentially have caused more hemodilution and transfusions. However, those amounts are difficult to interpret because data were sometimes missing and often incomplete, not taking into account the medications received. The concentration of saline used was variable, which affects the osmolality and proportion of fluid that remains intravascular. Also, the proportion of colloids was higher in the furosemide group and colloids remain in the intravascular space 3 times longer than a similar volume of crystalloids, causing more hemodilution. Accordingly, when these factors were accounted for, there was no difference in fluid volume between groups. This underlines the need for larger, prospective data collection and, ideally, randomized controlled trials.

Several other approaches to reduce PRBC transfusion in craniosynostosis surgery have been previously described. Studies have shown that erythropoietin administration before surgery can increase preoperative hemoglobin and thus reduce the likelihood of requiring a PRBC transfusion. ${ }^{8,12,16}$ Tranexamic acid has been shown to reduce intraoperative blood loss and transfusion needs in multiple settings, and recent studies have confirmed its effectiveness in craniosynostosis surgery. ${ }^{4,9,13}$ Other approaches in- 
clude autologous blood transfusion, ${ }^{3}$ preoperative hemodilution,,$^{16}$ and endoscopic surgery. ${ }^{15}$ The use of furosemide administration in relation to these alternative approaches remains to be determined.

\section{Conclusions}

The postoperative anemia observed in sagittal craniosynostosis surgery is in part due to excessive hemodilution and can be managed without resorting to PRBC transfusion. In this study, the postoperative transfusion rate was significantly reduced using postoperative furosemide administration to correct volemic status.

\section{References}

1. Boop FA, Chadduck WM, Shewmake K, Teo C: Outcome analysis of 85 patients undergoing the pi procedure for correction of sagittal synostosis. J Neurosurg 85:50-55, 1996

2. Breugem CC, van R Zeeman BJ: Retrospective study of nonsyndromic craniosynostosis treated over a 10 -year period. J Craniofac Surg 10:140-143, 1999

3. Carver ED, White N: Variations in autologous blood transfusion systems. J Craniofac Surg 20:580, 2009 (Letter)

4. Dadure C, Sauter M, Bringuier S, Bigorre M, Raux O, Rochette A, et al: Intraoperative tranexamic acid reduces blood transfusion in children undergoing craniosynostosis surgery: a randomized double-blind study. Anesthesiology 114:856861,2011

5. Duncan C, Richardson D, May P, Thiruchelvam J, Shong DC, Potter F, et al: Reducing blood loss in synostosis surgery: the Liverpool experience. J Craniofac Surg 19:1424-1430, 2008

6. Faberowski LW, Black S, Mickle JP: Blood loss and transfusion practice in the perioperative management of craniosynostosis repair. J Neurosurg Anesthesiol 11:167-172, 1999

7. Fearon JA, McLaughlin EB, Kolar JC: Sagittal craniosynostosis: surgical outcomes and long-term growth. Plast Reconstr Surg 117:532-541, 2006

8. Fearon JA, Weinthal J: The use of recombinant erythropoietin in the reduction of blood transfusion rates in craniosynostosis repair in infants and children. Plast Reconstr Surg 109:2190-2196, 2002

9. Goobie SM, Meier PM, Pereira LM, McGowan FX, Prescilla RP, Scharp LA, et al: Efficacy of tranexamic acid in pediatric craniosynostosis surgery: a double-blind, placebo-controlled trial. Anesthesiology 114:862-871, 2011

10. Gross JB: Estimating allowable blood loss: corrected for dilution. Anesthesiology 58:277-280, 1983

11. Hans P, Collin V, Bonhomme V, Damas F, Born JD, Lamy M: Evaluation of acute normovolemic hemodilution for surgical repair of craniosynostosis. J Neurosurg Anesthesiol 12:33-36, 2000

12. Helfaer MA, Carson BS, James CS, Gates J, Della-Lana D, Vander Kolk C: Increased hematocrit and decreased transfusion requirements in children given erythropoietin before undergoing craniofacial surgery. J Neurosurg 88:704-708, 1998

13. Holcomb JB: Tranexamic acid in elective craniosynostosis surgery: it works, but how? Anesthesiology 114:737-738, 2011

14. Istaphanous GK, Wheeler DS, Lisco SJ, Shander A: Red blood cell transfusion in critically ill children: a narrative review. Pediatr Crit Care Med 12:174-183, 2011

15. Jimenez DF, Barone CM: Endoscopic craniectomy for early surgical correction of sagittal craniosynostosis. J Neurosurg 88:77-81, 1998

16. Meneghini L, Zadra N, Aneloni V, Metrangolo S, Faggin R, Giusti F: Erythropoietin therapy and acute preoperative normovolaemic haemodilution in infants undergoing craniosynostosis surgery. Paediatr Anaesth 13:392-396, 2003

17. Meyer P, Renier D, Arnaud E: Intraoperative autologous blood transfusion in the surgical correction of craniosynostosis. Neurosurgery 39:213, 1996 (Letter)

18. Paut O, Lacroix F: Recent developments in the perioperative fluid management for the paediatric patient. Curr Opin Anaesthesiol 19:268-277, 2006

19. Seruya M, Oh AK, Boyajian MJ, Myseros JS, Yaun AL, Keating RF: Unreliability of intraoperative estimated blood loss in extended sagittal synostectomies. J Neurosurg Pediatr 8:443-449, 2011

20. Vega RA, Lyon C, Kierce JF, Tye GW, Ritter AM, Rhodes JL: Minimizing transfusion requirements for children undergoing craniosynostosis repair: the CHoR protocol. J Neurosurg Pediatr 14:190-195, 2014

21. Vergnaud E, Vecchione A, Blanot S, di Rocco F, Arnaud E, Renier D, et al: Reducing blood losses and transfusion requirements in craniosynostosis surgery: an endless quest? Anesthesiology 116:733-735, 2012

\section{Disclosure}

The authors report no conflict of interest concerning the materials or methods used in this study or the findings specified in this paper.

\section{Supplemental Information \\ Previous Presentation}

This work was presented in an oral presentation at the 42nd Annual Meeting of the AANS/CNS Section on Pediatric Neurological Surgery, Toronto, Ontario, Canada, December 6, 2013.

\section{Author Contributions}

Conception and design: Crevier, Harroud, Turgeon, Mercier. Acquisition of data: Harroud, Weil. Analysis and interpretation of data: Crevier, Harroud, Weil. Drafting the article: Harroud, Weil, Turgeon. Critically revising the article: all authors. Reviewed submitted version of manuscript: all authors. Approved the final version of the manuscript on behalf of all authors: Crevier. Statistical analysis: Crevier, Harroud. Administrative/technical/material support: Crevier. Study supervision: Crevier.

\section{Correspondence}

Louis Crevier, Section of Neurosurgery, CHU Sainte-Justine, University of Montreal, 3175 Chemin de la Côte-Sainte-Catherine, Montreal, QC H3T 1C5, Canada. email: louiscrevier@ hotmail.com. 